PSICOLOGÍA

IBEROAMERICANA
Psicología Iberoamericana ISSN: 1405-0943

revista.psicologia@ibero.mx

Universidad Iberoamericana, Ciudad de México México

\title{
Relación entre necesidades no satisfechas y sintomatología emocional en cuidadores de pacientes oncológicos
}

Sierra Murguía, Mariana; Martínez Bravo, Andrea; Padilla Rico, Amalia; Fraga Sastrías, Juan Manuel Relación entre necesidades no satisfechas y sintomatología emocional en cuidadores de pacientes oncológicos Psicología Iberoamericana, vol. 28, núm. 1, 2020

Universidad Iberoamericana, Ciudad de México, México

Disponible en: http://www.redalyc.org/articulo.oa?id=133963198005 
Psicología Iberoamericana, vol. 28, núm. 1,2020

Universidad Iberoamericana, Ciudad de México, México

Recepción: 03 Diciembre 2019 Aprobación: 23 Marzo 2020

Redalyc: http://www.redalyc.org/ articulo.oa?id=133963198005
Artículos

\section{Relación entre necesidades no satisfechas y sintomatología emocional en cuidadores de pacientes oncológicos}

Relationship between unmet needs and depressive, anxiety and burnout symptoms in oncologic primary informal caregivers

Mariana Sierra Murguía msierra@cancercentertec100.com Cáncer Center Tec 100, México

http://orcid.org/0000-0001-5806-0688

Andrea Martínez Bravo

Cáncer Center Tec 100, México

http://orcid.org/0000-0002-5856-7232

Amalia Padilla Rico

Cáncer Center Tec 100, México

http://orcid.org/0000-0002-5856-7232

Juan Manuel Fraga Sastrías

Cáncer Center Tec 100, México

http://orcid.org/0000-0002-9255-278X

Resumen: Existe un alto riesgo de sintomatología psicológica en los cuidadores primarios informales, esto se debe a la alta demanda física y emocional que conllevan sus tareas, llegando frecuentemente a la disminución de actividades de autocuidado, cambios en roles familiares, laborales y sociales, y al descuido de necesidades propias. El estudio aborda la relación entre necesidades insatisfechas y sintomatología emocional (depresión, ansiedad y sobrecarga de cuidador) en cuidadores primarios informales. El estudio es cuantitativo y transversal y se evaluaron 76 cuidadores primarios informales de pacientes oncológicos que se encontraban recibiendo tratamiento en una institución privada. Las características sociodemográficas de estos pacientes fueron $58 \%$ mujeres, con 49 años en promedio, $30 \%$ únicos cuidadores, $72 \%$ casados, $32.9 \%$ con escolaridad licenciatura. Se excluyeron cuidadores que no fueran exclusivos o que recibieran remuneración por las tareas de cuidado. Los instrumentos utilizados fueron el de Sobrecarga de Zarit, el Hospital Anxiety and Depression Scale (HADS), y el Support Person Unmet Needs Survey (SPUNS). Se hizo un análisis de regresión lineal múltiple para conocer la relación entre las necesidades descritas y la sintomatología emocional. El 27\% de la muestra tuvo ansiedad, el 15\% depresión, y el 12\% presentó sobrecarga de cuidador. Dentro de las necesidades identificadas, predominó la preocupación sobre el futuro de la enfermedad, seguido de necesidades personales. A su vez, las necesidades personales insatisfechas se asociaron de manera significativa a mayor ansiedad y depresión. Los cuidadores estudiados presentaron sintomatología emocional, la cual a su vez se asociaba con necesidades personales insatisfechas y a preocupaciones por el futuro. Se resalta la importancia de incluir a los cuidadores en la atención integral del paciente con cáncer; a identificar las necesidades desde el inicio del tratamiento, y a generar intervenciones enfocadas en la satisfacción de las mismas.

Palabras clave: necesidades de cuidadores, sobrecarga del cuidador, burnout, ansiedad, depresión, psicooncología, cuidador primario informal. 


\begin{abstract}
Primary informal caregivers have been described as a vulnerable population due to the high demand of tasks required in this role, leading to a decrease in self-care activities, changes in social, laboral and family roles, as well as a neglect of personal necessities. The study addresses the relation between burnout, anxiety, depression and needs in a group of primary caregivers of cancer patients. The study is quantitative and cross-sectional, and 76 primary caregivers of cancer patients were assessed. $58 \%$ of the caregivers are women, with an average age of 49 -years-old, $30 \%$ were the sole caregivers, $72 \%$ were married and $32.9 \%$ had a bachelors degree. Only primary caregivers who did not receive economic remuneration for their duties were included in the sample. Instruments used were Zarit Burnout Inventory, Hospital Anxiety and Depression Scale (HADS) and Support Person Unmet Needs Survey (SPUNS-SFM). Linear multiple regression analysis was used to examine the relationship between variables. In the study population, $27 \%$ presented with anxiety, $15 \%$ with depression and $12 \%$ with burnout symptomology. Respecting caregiver needs, concerns about disease progression and personal needs were predominant. These needs were also predictors of anxiety and depression. The importance of including caregivers in multidisciplinary care is justified, besides the psychologic evaluation, we suggest providing information about the future and disease, as well as self-care education to satisfy personal needs, because of its relationship with anxiety and depression.
\end{abstract}

Keywords: caregiver needs, caregiver overload, informal caregiver, burnout, anxiety, depression, psycho oncology.

\title{
Introducción
}

Los estándares de calidad en la atención han incrementado su importancia dentro del tratamiento oncológico. Un componente clave dentro de esta área, es la experiencia del paciente. En esta percepción en calidad de la atención, el papel del cuidador primario informal ha sido poco descrito, sin embargo resulta pertinente (Litzelman, Kent, Mollica, \& Rowland, 2016), ya que el bienestar percibido del paciente ha sido asociado directamente al de su cuidador resaltando la importancia de este rol (Ejem, Drentea, \& Clay, 2014). El cuidador primario informal, según la Sociedad Americana de Cáncer (American Cancer Society, 2019), es alguna persona que apoya a otra durante el proceso de la enfermedad de una manera no remunerada. Se le considera como miembro de una triada junto con el paciente y los profesionales de salud, ya que se involucran en la toma de decisiones relacionadas a la enfermedad; puede actuar como intermediario sobre la información entre el paciente y los especialistas, y brinda un sistema de apoyo al paciente (Lambert et al., 2013). El diagnóstico de cáncer y el tratamiento, crea una afectación psicológica para el paciente y también para su familia (Galindo Vázquez et al., 2015). En México, se han identificado algunos perfiles de los cuidadores, identificando como características más frecuentes, el sexo femenino, casada, con media de edad de 56-67 años, con parentesco de esposa o hija, y que por lo general no aceptan ayuda de otras personas (Ramírez \& Aguilar, 2009). Exclusivamente en población mexicana, se ha identifcado que una prevalencia de $31 \%$ de sobrecarga, con $76 \%$ de percepción de buena calidad de vida y una correlación positiva ligera entre estas variables (Valencia et al., 2017).

En los cuidadores de pacientes con cáncer se han descrito altos niveles de psicopatología y distrés (Northouse, Williams, Given, McCorkle, 
\& Netter, 2012), además de afectaciones físicas (Lund, Ross, Petersen, \& Groenvold, 2014; Park et al., 2013), las cuales pueden generarles incapacidad para mantener la participación en sus actividades e intereses, lo que se asocia a mayor disfunción psicosocial; por lo que el desarrollo y evaluación de intervenciones y programas destinados a los cuidadores en mantener sus actividades de autocuidado, deben tener un impacto positivo en cuanto a su bienestar emocional, pues resulta vital para mantener el cuidado a los pacientes a largo plazo (Park et al., 2013).

Dentro de las principales afectaciones reportadas en cuidadores primarios de pacientes oncológicos están las psicológicas, presentándose $67 \%$ con sintomatología depresiva leve y entre un $2 \%$ y $35 \%$ con sintomatología depresiva severa, así como un $40.7 \%$ de sintomatología ansiosa (Rangel-Domínguez et al., 2013). Estas cifras pueden aumentar cuando el paciente está en fase avanzada de la enfermedad, reportándose aproximadamente $13 \%$ cumpliendo criterios para un trastorno psiquiátrico (trastorno de pánico $8.0 \%$, trastorno depresivo mayor $4.5 \%$, trastorno de estrés post-traumático, $4.0 \%$, a $3.5 \%$ trastorno de ansiedad generalizada). Dentro de los síntomas físicos, $15 \%$ refieren dolor, 36.7\% trastornos del dormir, 33.3\% fatiga en la mañana y $30.3 \%$ fatiga general (Williams, Dixon, Feinn, \& McCorkle, 2015). De manera general, $25 \%$ de los cuidadores primarios informales, requieren atención de un servicio de salud mental.

En cuanto a variables identificadas como riesgo para los cuidadores, se describe en primer lugar el sexo, siendo el femenino quien tiene mayor probabilidad de presentar depresión, estrés, dificultades para adaptarse a la enfermedad y cambios de roles, mayores niveles de alteraciones en el sueño, dolor muscular y problemas para transportar al paciente, que el sexo masculino (Tang et al., 2013). La edad es otra variable que se ha reportado como riesgo, describiendo que a menor edad, se presentan mayores problemas financieros, dificultando el manejo del dolor del paciente (incrementándose el estrés); y mayor dificultad para continuar con sus actividades sociales que los cuidadores con mayor edad (mayores de 40 años) (generando mayor aislamiento y estrés).

El presentar necesidades insatisfechas, a su vez, también es factor de riesgo en esta población, ya que pueden presentarse sentimientos de "pérdida de uno/a mismo/a" y aislamiento. Cerca del 23\% de los cuidadores han referido no tener tiempo para atender sus propias necesidades, asociándose a un incremento en el malestar emocional (INSERO, 1995).

Como consecuencia de la demanda que puede sostener el ser un cuidador primario informal, y a las consecuencias emocionales que éste tiene, puede presentarse el síndrome de sobrecarga del cuidador, también conocido como claudicación física y emocional (Fundación para la prevención de riesgos laborales, 2005). Este síndrome suele estar provocado por las dificultades en el ajuste por el cambio radical de su modo de vida, el descuido de las propias necesidades y el desgaste que provoca ver cómo un ser querido va perdiendo progresivamente sus facultades físicas y psicológicas; pasando, conforme avanza el curso de la 
enfermedad, de ser un mero supervisor de las actividades del paciente, al que le procura los cuidados más básicos (Galindo Vázquez et al., 2015).

Las características más prevalentes del síndrome del cuidador primario son: trastornos en el patrón de sueño, irritabilidad, altos niveles de ansiedad, reacción exagerada a las críticas, dificultad en las relaciones interpersonales, sentimientos de desesperanza la mayor parte del tiempo, resentimiento hacia la persona que cuida, pensamientos de suicidio o de abandono, frecuentes dolores de cabeza o de espalda, pérdida de energía, sensación de cansancio y aislamiento (Zambrano Cruz \& Ceballos Cardona, 2007).

Este síndrome, por ende, constituye una situación estresante con peligro de desbordar y agotar los recursos y repercutir en la salud física del cuidador y su estado de ánimo (Herrera, Flórez, Romero, \& Montalvo, 2012).

Debido al riesgo psicosocial que implica ser un cuidador primario informal, se plantean las siguientes preguntas de investigación: ¿Cuáles son las necesidades de los cuidadores primarios informales de pacientes con cáncer tratados en un centro privado? ¿Cuál es la relación de estas necesidades con la ansiedad y depresión? ¿Cuál es la prevalencia de depresión, ansiedad y sobrecarga en esta muestra?

\section{Objetivos}

Conocer la relación entre las necesidades de los cuidadores (de información, preocupaciones sobre el futuro de la enfermedad del paciente, económicas, acceso de atención médica, personales y emocionales), con la ansiedad y depresión en cuidadores primarios informales de pacientes oncológicos tratados en un centro privado.

Describir los resultados de la evaluación realizada a un grupo de cuidadores primarios de pacientes oncológicos, en donde se aplicaron inventarios de sobrecarga del cuidador, ansiedad, depresión y necesidades.

\section{Método}

Es un estudio no experimental, cuantitativo y transversal. Se busca describir los resultados de las evaluaciones y conocer la relación entre las necesidades no satisfechas de los cuidadores, con la sobrecarga, ansiedad y depresión.

\section{Participantes}

Se incluyeron 76 cuidadores de pacientes oncológicos tratados en una institución privada, en la ciudad de Querétaro, México. Se les invitó a participar, de manera voluntaria, mientras sus familiares se encontraban en el centro recibiendo quimioterapia. El muestreo que se hizo fue por conveniencia, es decir, todos los cuidadores que aceptaran participar en un periodo entre julio de 2018 y julio de 2019. Estos fueron los criterios 
de selección que se consideraron: que fueran el cuidador primario (la persona que mayor tiempo dedicaba al cuidado del enfermo), que firmara el consentimiento informado, que fuera mayor de edad y que supiera leer y escribir. Se excluyó a los acompañantes que no fueran cuidador primario o que recibieran remuneración económica por los cuidados del paciente.

Variables Demográficas

Tabla 1

\section{Tabla 1}

Datos demográficos $(n=76)$

\begin{tabular}{|c|c|}
\hline Sexo femenino & $33(58 \%)$ \\
\hline Edad & 49 años, DE 8 \\
\hline Tiempo de cuidado (meses) & $\begin{array}{l}\text { Promedio } 4,7 \text { meses, rango 1.5-14 } \\
\text { DE } 3.1\end{array}$ \\
\hline Único cuidador $(n=53)$ & Sí: $23(30.3 \%)$ \\
\hline Estado Civil & $\begin{array}{l}\text { Casado } 55(72.4 \%) \\
\text { Solteros } 15(19.7 \%) \\
\text { Divorciado } 3(3.9 \%) \\
\text { Viudo } 3(3.9 \%)\end{array}$ \\
\hline Escolaridad & $\begin{array}{l}\text { Primaria } 6(7.9 \%) \\
\text { Secundaria } 4(5.3 \%) \\
\text { Preparatoria } 12(15.8 \%) \\
\text { Técnica } 8(10.5 \%) \\
\text { Licenciatura } 25(32.9 \%) \\
\text { Posgrado } 6(7.9 \%) \\
\text { No contestó } 15(19.7 \%)\end{array}$ \\
\hline Parentesco & $\begin{array}{l}\text { Hijo } 19(25 \%) \\
\text { Pareja } 25(32.9 \%) \\
\text { Padre/madre } 17(22.4 \%) \\
\text { Hermano } 7(9.2 \%) \\
\text { Otro } 8(10.5 \%)\end{array}$ \\
\hline
\end{tabular}

Nota: Para variables nominales, se presentan frecuencias y porcentajes, mientras que para variables de intervalo, media y desviación estándar.

\section{Datos demográficos $(n=76)$}

Nota: Para variables nominales, se presentan frecuencias y porcentajes, mientras que para variables de intervalo, media y desviación estándar.

\section{Instrumentos}

Los instrumentos utilizados fueron el de Sobrecarga de Zarit, el Hospital Anxiety and Depression Scale (HADS), y el Support Person Unmet Needs Survey (SPUNS-SFM). Las propiedades psicométricas se describen a continuación. 


\section{Escala de Ansiedad y Depresión Hospitalaria (Hospital Anxiety and Depression Scale) (HADS)}

Esta escala es un tamizaje para depresión y ansiedad. La versión original, desarrollada por Zigmond y Snaith (1983) consta de 14 reactivos, intercalando ítems para dos subescalas, una para ansiedad y otra para depresión. La versión mexicana, validada para pacientes oncológicos, consta de 12 reactivos que puntúan entre 0 y 3 . Para la calificación, se suman las puntuaciones nones (subescala de ansiedad), y de manera independiente, se suman las pares (subescala de depresión). Los puntos de corte sugeridos para esta población se obtuvieron a partir de un percentil mayor a 75 y se considera de 0-5 leve, 6-8 leve, 9-11 moderado, y mayor a 12 severo (las puntuaciones para depresión y para ansiedad se hacen de manera independiente). La escala cuenta con una consistencia interna de un Alpha de Cronbach total de .86; así como de .79 y .80 para cada subescala -ansiedad y depresión respectivamente- (Galindo et al., 2015).

\section{Inventario de Sobrecarga de Zarit (Zarit Burden Interview) (ZBI)}

El inventario Zarit es un inventario autoaplicado, que cuenta con 22 ítems del tipo Likert $(0-4)$ y se divide en 3 subescalas: impacto del cuidado, relaciones interpersonales, y expectativas sobre autoefectividad. Mide la percepción de carga del cuidado en el cuidador. En su versión original (Zarit \& Zarit, 1987), realizada en población de cuidadores no exclusivamente oncológicos, mostró un Alpha de Cronbach de .83 a .92. La versión mexicana, para cuidadores de pacientes oncológicos, cuenta con una consistencia interna de alfa de .90 y ha sido adaptada para su uso en cuidadores primarios de pacientes oncológicos mexicanos (GalindoVazquez et al., 2015).

\section{Support Person Unmet Need Survey (SPUNS-SFM)}

Versión original desarrollada en Canadá, consta de 26 ítems en 5 factores (Campbell et al., 2014). La versión adaptada para población mexicana consta de 23 ítems con 6 factores: preocupaciones sobre el futuro, acceso a los sistemas de salud, información, trabajo y finanzas, necesidades personales y emocionales. Los 23 ítems son tipo Likert, se puntúan de 0 a 4 , y se suman los puntajes por subescala y otra general. A mayor puntuación, mayor será la necesidad. Muestra consistencia interna de Alfa de Cronbach de .70 a .88 entre los diferentes factores. También mostró validez de contenido mediante criterio de expertos, validez de constructo mediante un análisis factorial exploratorio, validez de convergencia mediante una correlación del spuns-sfm con escalas de calidad de vida (mos sf12), ansiedad y depresión (escala de ansiedad y depresión hospitalaria hads); mostró confiabilidad de test retest utilizando coeficiente de correlación intraclase. Este concluyó que sus propiedades psicométricas son adecuadas para uso en cuidadores 
de pacientes oncológicos mexicanos (Doubova, Aguirre-Hernandez, Infante-Castañeda, Martinez-Vega, \& Pérez-Cuevas, 2015).

\section{Procedimiento}

Al estar el paciente oncológico recibiendo quimioterapia, se invitaba al cuidador primario al área de psico-oncología para realizar la evaluación. Se le explicaban los objetivos del estudio y se le indicaba que su participación era confidencial y voluntaria. Los participantes contestaron los inventarios, que posteriormente fueron capturados en una base de datos para su análisis estadístico utilizando el programa spss.

\section{Análisis de datos}

Se utilizó estadística descriptiva para variables demográficas (sexo, edad, escolaridad, parentesco) y resultados generales de los inventarios (HADS, Zarit, SPUNS-SFM). Posteriormente se hizo correlación de Pearson para conocer la relación entre las necesidades de los cuidadores con ansiedad y depresión. Por último se hizo un análisis de regresión lineal múltiple para conocer la relación entre las necesidades de los cuidadores con depresión y ansiedad.

\section{Resultados}

\section{Resultados Generales de la Evaluación}

En cuanto a los resultados obtenidos por la Escala de Ansiedad y Depresión Hospitalaria (HADS), se describen los resultados en cuanto a prevalencia de ansiedad y depresión en la Tabla 2.

Tabla 2

\section{Tabla 2}

Resultados de Escala de Ansiedad y Depresión Hospitalaria (HADS)

\begin{tabular}{lllll} 
& \multicolumn{2}{l}{ Nivel de Ansiedad } & \multicolumn{2}{c}{ Nivel de Depresión } \\
& $\mathrm{n}$ & $\%$ & $\mathrm{n}$ & $\%$ \\
\cline { 2 - 5 } No presenta & 56 & 73.7 & 64 & 84.2 \\
Leve & 13 & 16 & 11 & 14.5 \\
Moderada & 5 & 7 & 1 & 1.3 \\
Grave & 1 & 2 & - & - \\
TOTAL & 76 & 100 & 76 & 100
\end{tabular}


Con relación a la prevalencia de sobrecarga del cuidador, evaluada con el Inventario de Sobrecarga de Zarit, se observó que el $88.2 \%$ no experimenta sobrecarga; mientras el $12 \%$ restante perciben la carga entre leve e intensa (Tabla 3).

Tabla 3

Tabla 3

Resultados de Inventario de Sobrecarga de Zarit

\begin{tabular}{lll} 
& $\mathrm{n}$ & $\%$ \\
\cline { 2 - 3 } No presenta & 67 & 88.2 \\
Leve & 6 & 7.9 \\
Intensa & 3 & 3.9 \\
TOTAL & 57 & 100
\end{tabular}

Resultados de Inventario de Sobrecarga de Zarit

Las evaluación de las necesidades de los cuidadores mostró una mayor frecuencia en preocupaciones sobre el futuro, seguida de necesidades personales, emocionales y de información. En la Tabla 3 se muestran los porcentajes de necesidades que presentaban los cuidadores de acuerdo con el instrumento Support Person Unmet Need Survey (SPUNS-SFM).

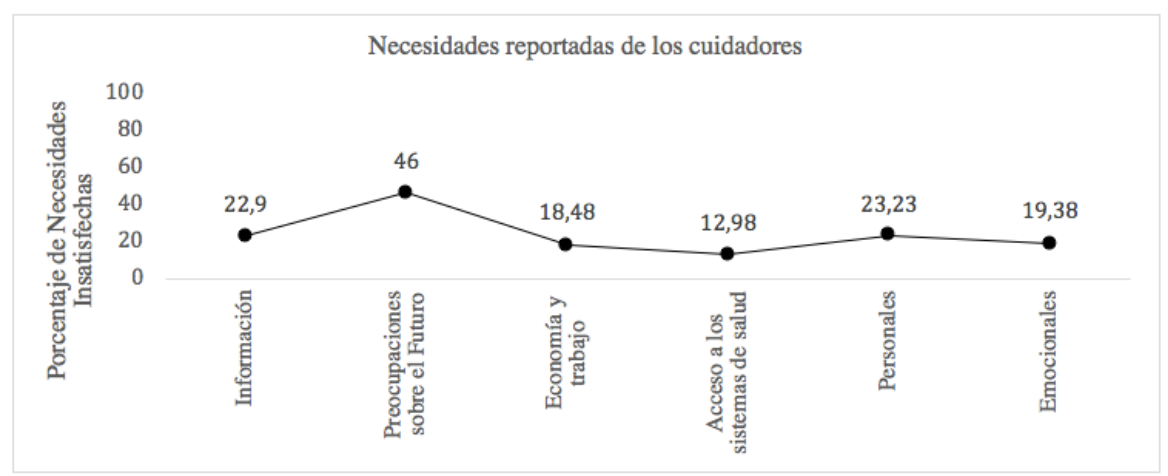

Figura 1. Necesidades insatisfechas del cuidador (SPUNS-SFM).

Figura 1. Necesidades insatisfechas del cuidador (spuns-sfmspuns-sfm).

Más adelante se realizó un análisis para conocer correlaciones entre las necesidades insatisfechas, la ansiedad y depresión de los cuidadores, para lo cual se sacó la correlación de Spearman, mostrando las correlaciones significativas que se describen en la Tabla 4. 
Tabla 4

Tabla 4

Correlaciones entre necesidades insatisfechas y niveles de ansiedad y depresión de los cuidadores

HADSA HADSD Información Futuro Económicas Acceso Personal emocional

\begin{tabular}{|c|c|c|c|c|c|c|c|c|c|}
\hline & & & 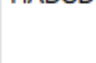 & 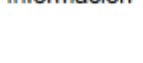 & & 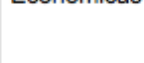 & $\begin{array}{l}\text { a } \\
\text { atención }\end{array}$ & & \\
\hline \multirow[t]{3}{*}{ HADSA } & $\begin{array}{l}\text { Correlación } \\
\text { de Pearson }\end{array}$ & 1 & $362^{*}$ & $278^{\circ}$ & $347^{*}$ & , 160 &, $264^{\circ}$ &, $599^{* \prime}$ & $428^{\prime \prime}$ \\
\hline & $\begin{array}{l}\text { Sig. } \\
\text { (bilateral) }\end{array}$ & &, 001 & ,024 & ,004 & ,200 & ,032 &, 000 &, 000 \\
\hline & $\mathrm{N}$ & 75 & 75 & 66 & 66 & 66 & 66 & 66 & 66 \\
\hline \multirow[t]{3}{*}{ HADSD } & $\begin{array}{l}\text { Correlación } \\
\text { de Pearson }\end{array}$ &, $362^{*}$ & 1 & $283^{\circ}$ & $356^{*}$ & ,172 & ,223 & $488^{*}$ &, $393^{*}$ \\
\hline & $\begin{array}{l}\text { Sig. } \\
\text { (bilateral) }\end{array}$ & ,001 & & ,021 &, 003 & 168 & ,072 & ,000 & ,001 \\
\hline & $\mathrm{N}$ & 75 & 75 & 66 & 66 & 66 & 66 & 66 & 66 \\
\hline \multirow[t]{3}{*}{ Información } & $\begin{array}{l}\text { Correlación } \\
\text { de Pearson }\end{array}$ &, $278^{\circ}$ & $283^{\circ}$ & 1 &, $721^{*}$ &, $528^{*}$ & ,671" & $390^{*}$ &, $580^{\circ}$ \\
\hline & $\begin{array}{l}\text { Sig. } \\
\text { (bilateral) }\end{array}$ &, 024 &, 021 & & 000 &, 000 &, 000 &, 001 & 000 \\
\hline & $\mathrm{N}$ & 66 & 66 & 67 & 67 & 67 & 67 & 67 & 67 \\
\hline \multirow[t]{3}{*}{ Futuro } & $\begin{array}{l}\text { Correlación } \\
\text { de Pearson }\end{array}$ &, $347^{*}$ &, $356^{*}$ &, $721^{*}$ & 1 &, $356^{*}$ & $456^{*}$ &, $511^{*}$ & $628^{\circ}$ \\
\hline & $\begin{array}{l}\text { Sig. } \\
\text { (bilateral) }\end{array}$ &, 004 &, 003 &, 000 & &, 003 &, 000 &, 000 & 000 \\
\hline & $\mathrm{N}$ & 66 & 66 & 67 & 67 & 67 & 67 & 67 & 67 \\
\hline \multirow[t]{3}{*}{ Económicas } & $\begin{array}{l}\text { Correlación } \\
\text { de Pearson }\end{array}$ & 160 & 172 &, $528^{*}$ &, $356^{*}$ & 1 & $659^{\prime \prime}$ &, $264^{\circ}$ & $388^{*}$ \\
\hline & $\begin{array}{l}\text { Sig. } \\
\text { (bilateral) }\end{array}$ & ,200 & , 168 &, 000 &, 003 & &, 000 &, 031 &, 001 \\
\hline & $\mathrm{N}$ & 66 & 66 & 67 & 67 & 67 & 67 & 67 & 67 \\
\hline \multirow[t]{3}{*}{$\begin{array}{l}\text { Acceso a } \\
\text { atención }\end{array}$} & $\begin{array}{l}\text { Correlación } \\
\text { de Pearson }\end{array}$ & $264^{*}$ &, 223 &, $671^{*}$ & $456^{\circ}$ & $659^{*}$ & 1 & $400^{\circ}$ &, $581^{\prime \prime}$ \\
\hline & $\begin{array}{l}\text { Sig. } \\
\text { (bilateral) }\end{array}$ &, 032 &, 072 &, 000 &, 000 &, 000 & & 001 &, 000 \\
\hline & $\mathrm{N}$ & 66 & 66 & 67 & 67 & 67 & 67 & 67 & 67 \\
\hline \multirow[t]{3}{*}{ Personal } & $\begin{array}{l}\text { Correlación } \\
\text { de Pearson }\end{array}$ &, $599^{*}$ & $488^{\circ}$ & $390^{*}$ &, $511^{*}$ & $264^{\circ}$ &, $400^{*}$ & 1 & $648^{*}$ \\
\hline & $\begin{array}{l}\text { Sig. } \\
\text { (bilateral) }\end{array}$ &, 000 &, 000 & ,001 &, 000 &, 031 & 001 & &, 000 \\
\hline & $\mathrm{N}$ & 66 & 66 & 67 & 67 & 67 & 67 & 67 & 67 \\
\hline \multirow[t]{3}{*}{ Emocional } & $\begin{array}{l}\text { Correlación } \\
\text { de Pearson }\end{array}$ & $428^{\circ}$ & $393^{*}$ &, $580^{\circ}$ & $628^{*}$ & $388^{*}$ &, $581^{*}$ & $648^{\circ}$ & 1 \\
\hline & $\begin{array}{l}\text { Sig. } \\
\text { (bilateral) }\end{array}$ &, 000 &, 001 &, 000 &, 000 &, 001 &, 000 &, 000 & \\
\hline & $\mathrm{N}$ & 66 & 66 & 67 & 67 & 67 & 67 & 67 & 67 \\
\hline
\end{tabular}

Notas: **. La correlación es significativa en el nivel 0,01 (bilateral).

*. La correlación es significativa en el nivel 0,05 (bilateral).

Correlaciones entre necesidades insatisfechas y niveles de ansiedad y depresión de los cuidadores

Se identifican correlaciones positivas significativas medias, entre niveles de ansiedad personales $(r=.599 \mathrm{p}<.000)$ y emocionales $(\mathrm{r}=.428 \mathrm{p}<.000)$. En cuanto a la depresión, correlacionó positivamente y significativamente con necesidades personales $(\mathrm{r}=.488 \mathrm{p}<.000)$.

Por último, se hizo un análisis de regresión lineal múltiple para conocer la relación entre las variables que mostraron mayor correlación. Se identificaron las necesidades personales insatisfechas como predictoras de depresión (R .488; R2 .239; F 20.05; p<.000) (gráfico 2); y de ansiedad (R.599; R2.35; F 35.88; p<.000) (gráfico 3). 


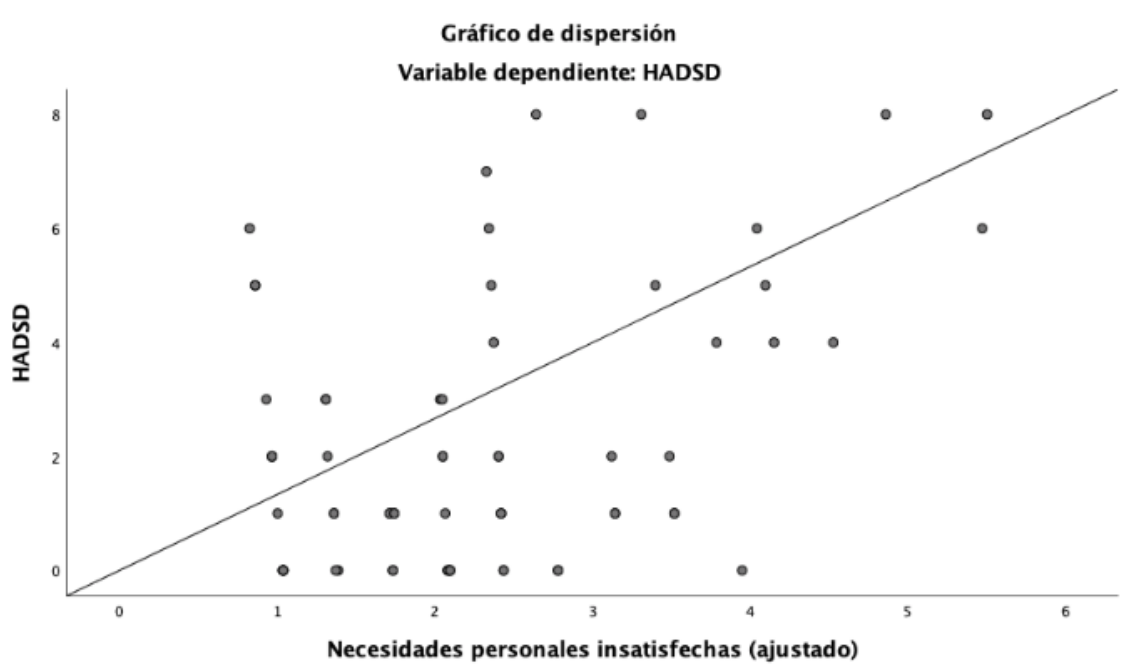

Figura 2. Relación entre HADS D (depresión) y necesidades personales insatisfechas.

Figura 2. Relación entre HADS D (depresión) y necesidades personales insatisfechas.

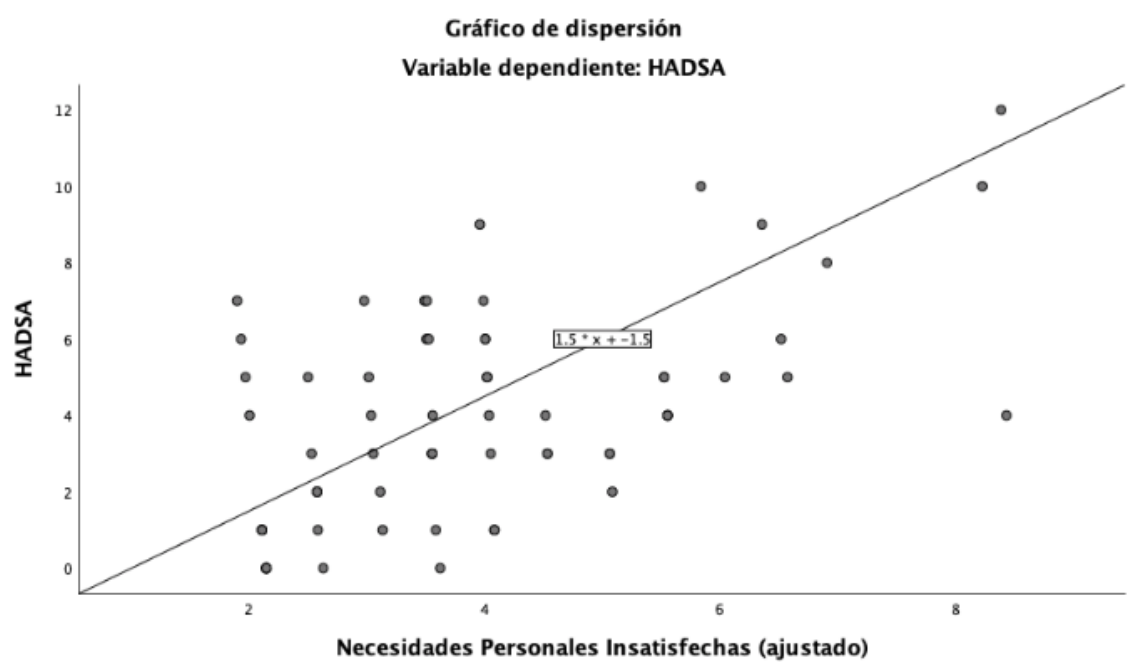

Figura 3. Relación entre HADS A (ansiedad) y necesidades personales insatisfechas.

Figura 3. Relación entre HADS A (ansiedad) y necesidades personales insatisfechas.

\section{Discusión}

El cáncer no sólo afecta al paciente, sino al cuidador primario informal, quien debe involucrarse en diferentes tareas relacionadas al tratamiento y enfermedad de la persona a su cargo. Todo esto conlleva a una serie de consecuencias emocionales, por lo que es importante su evaluación y tratamiento oportuno con el objetivo de mantener una adecuada adherencia terapéutica y promover el apoyo social (Martínez Ruiz, Díaz Vega, \& Gómez Blanco, 2010). El bienestar del cuidador primario informal ha sido asociado con la percepción de bienestar y calidad de la atención en el paciente oncológico (Ejem et al., 2014; Litzelman 
et al., 2016), por lo que se justifica incluirlo en la atención integral del paciente, identificando las necesidades de los mismos. En cuanto a las necesidades identificadas en esta muestra, la más prevalente era la preocupación por el futuro de la enfermedad, seguida por necesidades personales insatisfechas (autocuidado). Se identificó una relación media entre necesidades personales insatisfechas, con ansiedad y depresión. Las necesidades personales se refieren a la práctica de actividades que los individuos inician y realizan por su propia cuenta para mantener su salud y bienestar (Dionne-Odom et al., 2017). Al estar insatisfecha esta necesidad, según los resultados del estudio, los cuidadores dejan de realizar estas actividades de autocuidado, quedando más vulnerables a consecuencias emocionales como ansiedad o depresión

Dentro de las consecuencias emocionales que implica ser un cuidador primario, las más reportadas han sido sobrecarga de cuidador, depresión y ansiedad (Martínez Ruiz et al., 2010). En el presente estudio, se analizaron sus prevalencias y la relación que tenían con las necesidades insatisfechas que reportaba el grupo de cuidadores evaluados. En cuanto a la depresión, en la evaluación realizada con el HADS, 16\% de los cuidadores presentaba algún grado de la misma, mientras que para ansiedad fue el $27 \%$. Esto coincide con lo reportado en la literatura de la investigación sobre el nivel de depresión de los cuidadores primarios en México (CarreónNieto, Pérez Negrón- Mata, \& Olague-Urincho, 2014), reforzando así la importancia de una adecuada evaluación e intervención.

En un estudio realizado por Litzelman et al. (2016), se asoció el estado emocional del cuidador con la percepción de la calidad de la atención en pacientes con cáncer de colon y pulmón, que pertenecían al grupo de pacientes del Cancer Care Outcomes Research and Surveillance (CanCORS), en Estados Unidos. Las evaluaciones de los cuidadores se hicieron entre 5 y 6 meses después del diagnóstico oncológico, y las de los pacientes entre 4 y 5 meses post diagnóstico. Se identificó que por cada punto aumentado de sintomatología depresiva, los pacientes tendían a disminuir un 6\% su percepción de calidad de vida. En el mismo estudio, cuando los cuidadores reportaban pobre autocuidado, los pacientes tendían a reportar cuatro veces menos calidad de vida. Se concluye así, que la salud mental del cuidador se encuentra relacionada a la salud mental, al ajuste a la enfermedad y a la calidad de vida del paciente; resaltando la importancia de la atención al cuidador primario informal en el tratamiento oncológico. En el presente estudio se identificó una relación significativa entre las necesidades insatisfechas con la ansiedad y depresión en el grupo de cuidadores, por lo que resulta relevante evaluar y atender estas necesidades, con la intención de promover la salud mental de este grupo.

Conocer esta relación permite realizar intervenciones encaminadas a satisfacer las necesidades de los cuidadores y se sugiere como línea de investigación conocer si la satisfacción de estas necesidades de manera oportuna, ayuda a disminuir el riesgo para desarrollar ansiedad, depresión y sobrecarga en esta población. 
Dentro de las limitaciones del estudio, se encuentra que el tiempo que los cuidadores tienen haciendo estas tareas es variado, y se perdió en un $30 \%$ de la muestra, por lo que no se incluyó en los análisis, tampoco se identifica el tiempo que dedican por semana y qué tanto ha interferido con sus actividades habituales (variables que pueden relacionarse con sintomatología de ansiedad, depresión y sobrecarga); por lo cual se sugiere evaluarlo en futuras investigaciones.

Así mismo, resultaría conveniente implementar intervenciones encaminadas a modificar estas preocupaciones mediante psicoeducación y brindando información sobre el futuro y estrategias de autocuidado para prevención de la sobrecarga del cuidador, así como investigar si esto tiene impacto en la ansiedad y sobrecarga de los cuidadores.

\section{Conclusión}

Aproximadamente $20 \%$ de los cuidadores presentaron algún tipo de afectación en el estado anímico, por lo que se resalta la importancia de su evaluación oportuna, e intervención psicológica específica acorde con sus necesidades.

Dentro de las preocupaciones presentadas en la muestra, cerca de la mitad presentó preocupaciones por el futuro de la enfermedad, seguido por necesidades personales insatisfechas y necesidades de información.

Existe una relación significativa entre necesidades personales insatisfechas, ansiedad y depresión. Por ende, se sugiere investigar la relación más directa entre las variables, y brindar estrategias que cubran estas necesidades, como talleres psicoeducativos e informativos, además de conocer si esto tiene impacto en la prevalencia de ansiedad y depresión en esta población; a su vez, conocer si esto impacta la calidad de vida del paciente oncológico.

\section{Referencias}

American Cancer Society. (2019). Caregiver resource guide. Recuperado de http s://www.cancer.org/treatment/caregivers.html

Campbell, S. H., Carey, M., Sanson-Fisher, R., Barker, D., Turner, D., TaylorBrown, J., \& Hall, A. (2014). Measuring the unmet supportive care needs of cancer support persons: The development of the Support Person's Unmet Needs Survey-short form. Europearn Journal of Cancer Care, 23(2), 255-262. https://doi.org/10.1111/ecc.12138

Carreón-Nieto, C. H., Negrón-Mata, E. P., \& Olague-Urincho, V. E. (2014). Nivel de depresión en el cuidador primario del paciente con cáncer. Revista de Enfermería del Instituto Mexicano del Seguro Social, 22(3), 131-134.

Dionne-Odom, J. N., Demark-Wahnefried, W., Taylor, R. A., Rocque, G. B., Azuero, A., Acemgil, A., ... \& Heaton, K. (2017). The self-care practices of family caregivers of persons with poor prognosis cancer: Differences by varying levels of caregiver well-being and preparedness. Supportive Care in Cancer, 25(8), 2437-2444. https://doi.org/10.1007/s00520-017-3650-7 
Doubova, S. V., Aguirre-Hernandez, R., Infante-Castañeda, C., Martinez-Vega, I., \& Pérez-Cuevas, R. (2015). Needs of caregivers of cancer patients: Validation of the Mexican version of the Support Person Unmet Needs Survey (SPUNS-SFM). Supportive Care in Cancer, 23(10), 2925-2935. h ttps://doi.org/10.1007/s00520-015-2658-0

Ejem, D. B., Drentea, P., \& Clay, O. J. (2014). The effects of caregiver emotional stress on the depressive symptomatology of the care recipient. Aging Mental Health, 19(1), 55-62. https://doi.org/10.1080/13607863.2014. 915919

Fundación para la prevención de riesgos laborales. (2005). Boletines para la prevención de riesgos laborales. Recuperado de http://portal.ugt.org/saludlaboral/publicaciones_new/files_boletines 2005/tc\%20boletines\%202005.pdf

Galindo Vázquez, O., Rojas Castillo, E., Ascencio Huertas, L., Meneses García, A., Aguilar-Ponce, J. L., Olvera Manzanilla, E., ... Alvarado-Aguilar, S. (2015). Guía de práctica clínica para la atención psico-oncológica del cuidador primario informal de pacientes con cáncer. Psicooncología, 12(1), 87-104. https://doi.org/10.5209/rev

Galindo-Vazquez, O., Benjet, C., Cruz-Nieto, M. H., Rojas-Castillo, E., Riveros-Rosas, A., Meneses-Garcia, A., ... Alvarado-Aguilar, S. (2015). Psychometric properties of the Zarit Burden Interview in Mexican caregivers of cancer patients. Psycho-Oncology, 24(5), 612-615. https://d oi.org/10.1002/pon.3686

Galindo, O., Benjet, C., Juárez García, F., Rojas, E., Riveros, A., Aguilar, J. L., ... Alvarado, S. (2015). Propiedades psicométricas de la Escala Hospitalaria de Ansiedad y Depresión (HADS) en una población de pacientes oncológicos mexicanos. Salud Mental, 38(4), 253-258. https:/ /doi.org/10.17711/SM.0185-3325.2015.035

Herrera, A., Flórez, I. E., Romero, E., \& Montalvo, A. (2012). Soporte social a cuidadores familiares de personas con enfermedad crónica en Cartagena. Aquichan, 12(3), 286-297. https://doi.org/10.5294/aqui.2012.12.3.7

INSERSO. (1995). Cuidados en la vejez. El apoyo informal. INSERSO.

Lambert, S. D., Girgis, A., McElduff, P., Turner, J., Levesque, J. V, Kayser, K., ... Barker, D. (2013). A parallel-group, randomised controlled trial of a multimedia, self-directed, coping skills training intervention for patients with cancer and their partners: Design and rationale. BMJ Open, 3(7), e003337. http://dx.doi.org/10.1136/bmjopen-2013-003337

Litzelman, K., Kent, E. E., Mollica, M., \& Rowland, J. H. (2016). How does caregiver well-being relate to perceived quality of care in patients with cancer? Exploring associations and pathways. Journal of Clinical Oncology, 34(29), 3554-3561. https://doi.org/10.1200/JCO.2016.67.3434

Lund, L., Ross, L., Petersen, M. A., \& Groenvold, M. (2014). Cancer caregiving tasks and consequences and their associations with caregiver status and the caregiver's relationship to the patient: A survey. BMC Cancer, 14(1), 541. https://doi.org/10.1186/1471-2407-14-541

Martínez Ruiz, E. D. C., Díaz Vega, F. E., \& Gómez Blanco, E. I. (2010). Sobrecarga del cuidador primario que asiste a un familiar con cáncer. Universidad Veracruzana Comité Editorial Ampliado Comité Ecuatorial $I U, 6(11), 32-39$. 
Northouse, L., Williams, A. L., Given, B., McCorkle, R., \& Netter, F. H. (2012). Psychosocial care for family caregivers of patients with cancer. Journal of Clinical Oncology, 30(11), 1227-1234. https://doi.org/10.1200/JCO.20 11.39 .5798

Park, B., Kim, S. Y., Shin, J. Y., Sanson-Fisher, R. W., Shin, D. W., Cho, J., \& Park, J. H. (2013). Prevalence and predictors of anxiety and depression among family caregivers of cancer patients: A nationwide survey of patient-family caregiver dyads in Korea. Supportive Care in Cancer, 21(10), 2799-2807. https://doi.org/10.1007/s00520-013-1852 $-1$

Ramírez, B. S. B., \& Aguilar, S. A. (2009). Desgaste físico y emocional del cuidador primario en cáncer. Cancerología, 4, 39-46.

Rangel-Domínguez, N. E., Ascencio-Huertas, L., Ornelas-Mejorada, R. R., Allende-Pérez, S. R., Landa-Ramírez, E., \& Sánchez-Sosa, J. J. (2013). Efectos de la solución de problemas sobre los comportamientos de autocuidado de cuidadores de pacientes oncológicos en fase paliativa: un estudio piloto. Psicooncología, 10(2/3), 365-376. https://doi.org/10.520 9/rev_PSIC.2013.v10.n2-3.43455

Tang, S. T., Chang, W. C., Chen, J. S., Wang, H. M., Shen, W. C., Li, C. Y., \& Liao, Y. C. (2013). Course and predictors of depressive symptoms among family caregivers of terminally ill cancer patients until their death. Psycho\# Oncology, 22(6), 1312-1318. https://doi.org/10.1002/pon.3141

Valencia, M.C., Meza-Osnaya, G., Pérez Cruz, I., Cortes-Campero, N., Hernández Ovalle, J., Hernández- Paredes, P., Juárez Romero, K., Chino Hernández B., \& Romero-Figueroa, M.S. (2017). Factores que intervienen en la sobrecarga del cuidador primario del paciente con cáncer. Revista de Calidad Asistencial, 32(4), 221-225. https://doi.org/10.1016/ j.cali.2016.11.003

Williams, A. L., Dixon, J., Feinn, R., \& McCorkle, R. (2015). Cancer family caregiver depression: Are re 1 ig ion - related variables important? Psycho\#Oncology, 24(7), 825-831. https://doi.org/10.1002/pon.3647

Zambrano Cruz, R., \& Ceballos Cardona, P. (2007). Síndrome de carga del cuidador. Revista Colombiana de Psiquiatría, 36(1), 26-39.

Zarit, S. H., \& Zarit, J. M. (1987). The memory and behavior problems checklist: $1987 R$ and the burden interview (technical report). Pennsylvania State University.

Zigmond, A. S., \& Snaith, R. P. (1983). The hospital anxiety and depression scale. Acta Psychiatrica Scandinavica, 67(6), 361-370. https://doi.org/10. 1111/j.1600-0447.1983.tb09716.x 